\title{
How do the Swiss produce their New National Map Series?
}

\author{
Mark Wigley \\ Esri Switzerland Ltd.
}

Keywords: Generalisation, Map Design, Map Production, Topographic Mapping,

Transition to Modern and Contemporary Mappings.

\begin{abstract}
:
Switzerland, not unlike Japan, has a difficult and challenging topography to map.

The Swiss have been officially mapping their country now for nearly 175 years, which started when the "Bureau topographique fédéral" first published the initial set of official National maps in 1845. The various Swiss National Map series have been revised a number of times throughout its history and the latest revision and update was carried out in 2014 when the Federal Office of Topography swisstopo, published the first four sheets in the new generation of maps at 1:25'000.
\end{abstract}

Swisstopo have, with their new revised map series not only reviewed, challenged and changed their map production procedures but completely redesigned them.

The new production procedures for the national map series 1:10'000, 1:25'000, 1:50'000 and 1:100'000 are now all based on the 1:10'000 Topographic Landscape Model (TLM) where GIS methodology and software have replaced the old cartographic software.

Each of these four scales brings its own unique series of challenges such as generalisation, map design and labelling, and each goes through the same three main production procedures: automatic generalisation, cartographic improvement and manual cartographic finishing.

The automatic model and cartographic generalisation are performed by a large series of procedures, taking the base TLM data and preparing it for the required scale.

The cartographic improvement involves running preconfigured, so-called "Carto Processes" which perform cartographic improvements such as improving the road endings, creating annotations and various masks, rotating symbols and suppressing certain feature details.

The manual cartographic finishing (not required for the fully automatic 1:10'000 national map) is carried out by a highly skilled cartographic workforce, which take over where the automatic procedures fail to produce the required cartographic finish which the local market still demands from its National Mapping Agency. 